\title{
Acute Motor Axonal Neuropathy Improvement 20 Days After Hyperbaric Oxygen Therapy
}

This article was published in the following Dove Press journal:

International Medical Case Reports Journal

\author{
Ni Komang Sri Dewi \\ Untari (D) ${ }^{1,2}$ \\ Kurnia Kusumastuti (iD ${ }^{3}$ \\ Guritno Suryokusumo iD ${ }^{4}$ \\ I Ketut Sudiana ${ }^{5}$ \\ 'Department of Hyperbaric, Drs. Med. \\ Rijadi S. Phys. Naval Health Institute, \\ Surabaya, Indonesia; ${ }^{2}$ Doctoral Program, \\ Faculty of Medicine, Airlangga University, \\ Surabaya, Indonesia; ${ }^{3}$ Department of \\ Neurology, Faculty of Medicine, Airlangga \\ University, Surabaya, Indonesia; \\ ${ }^{4}$ Department of Hyperbaric, Faculty of \\ Medicine, Pembangunan Nasional \\ University, Jakarta, Indonesia; \\ ${ }^{5}$ Department of Pathology Anatomy, \\ Faculty of Medicine, Airlangga University, \\ Surabaya, Indonesia
}

Correspondence: Ni Komang Sri Dewi Untari

Department of Research and Development, Drs. Med. R. Rijadi S., Phys. Naval Health Institute, Surabaya, Indonesia

Email komang_neuro@yahoo.com

\begin{abstract}
We reported a rare case demonstrating that the hyperbaric oxygen chamber provided faster clinical improvement in a patient with a variant of Guillain-Barre Syndrome (GBS). A patient with progressive, acute weakness of upper extremity locomotor muscles and with difficulty breathing and swallowing was diagnosed with axonal GBS. Despite life-saving conventional therapies, there was no significant improvement until day 5. During hyperbaric oxygen therapy, there were daily gradual improvements until day 20, at which time the patient was capable of walking slowly without using a walking aid.
\end{abstract}

Keywords: acute motor axonal neuropathy, hyperbaric oxygen

\section{Introduction}

Guillain-Barre syndrome is an acute generalized polyneuropathy affecting 1 to 2 out of 100,000 people per year. ${ }^{1}$ Acute Motor Axonal Neuropathy (AMAN) is characterized by electrophysiologic and pathologic evidence of primary axonal degeneration. ${ }^{2}$ Limb weakness is among the main causes of degeneration of axons and may occur after Guillain-Barre syndrome, in which the antibody binds to the gangliosides. This condition may cause a 50 severe neurological disability, urgently requiring intravenous immunoglobulin (IVIG). However, there was no significant improvement. We note the recently published report of a patient with refractory AMAN who responded positively after initiation of $\mathrm{HBOT}^{3}$ and here we present another case with a positive outcome. In this report, a patient with tetraparesis showed motor improvements after hyperbaric oxygen therapy (HBOT).

\section{Case Report}

A 36-year-old woman presenting to the hyperbaric center with a history of weakness of all limbs was diagnosed with GBS. She had been in the ICU for eight days and received IVIG therapy for five days. Despite the conventional treatment, there was no improvement. She did not receive the second session of IVIG therapy. This patient had been discharged for two days before seeking hyperbaric therapy.

At the hyperbaric center, we found the woman in a wheelchair with steady vital signs and general muscle pain. Sensory examination showed hypoesthesia $(+1)$ of both extremities. Deep tendon reflexes (DTR) of upper and lower extremities were reduced $(+1)$. The upper limb muscle strength according to Medical Research Council (MRC) scale was 1 at proximal side and 2 at distal side. The limb muscle strength was also 1 at proximal side and 2 at distal side (Figure 1). Laboratory results were as follows: WBC: 


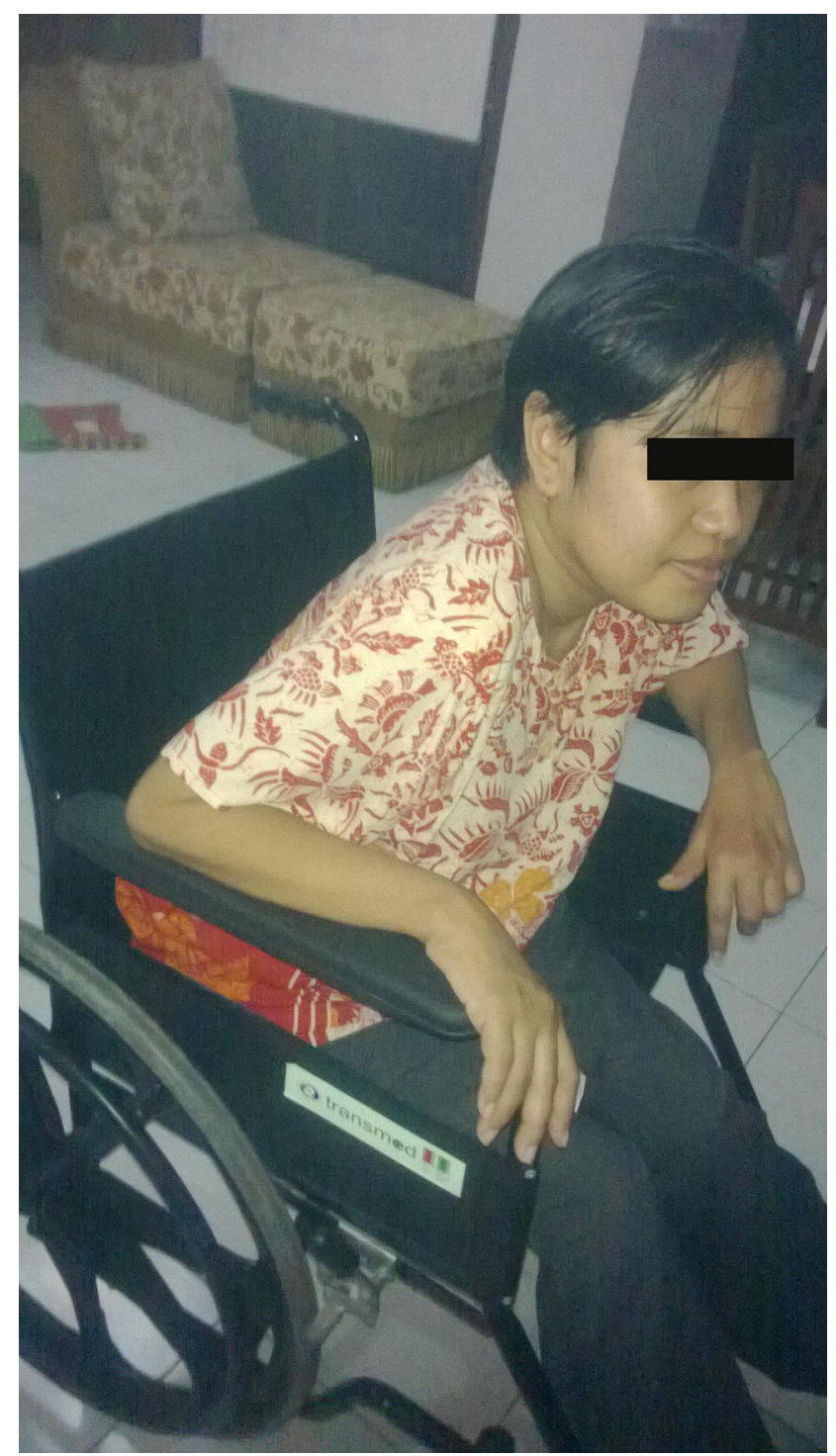

Figure I A woman, thirty-six years old, diagnosed with an axonal Guillain-Barre syndrome after IVimmunoglobulin treatment.

9000; lymphocytes: 60\%; PMN: 40\%; Hb: 11; CRP: +1; CSF examination: WBC: 0; RBC: 0; protein: 53; glucose: 82 . Electromyography examination resulted in axonal GuillainBarre syndrome diagnosis.

The patient underwent HBOT with a pressure of 2.4 atmospheres (ATA), administration of three 30-min cycles of $100 \%$ oxygen via a mask, and ten 5 -min periods of resting. After resting for two days, the patient returned to undergo HBOT for ten days. On day 1, she began to move her fingers slightly. On day 2 , she was able to slightly bend her wrists and move her toes (Figure 2).

At this time, with the slight motor improvement, she was able to sleep 6 hours at night, began to smile, and was more willing to eat. She started to be able to bend her arms on day 5

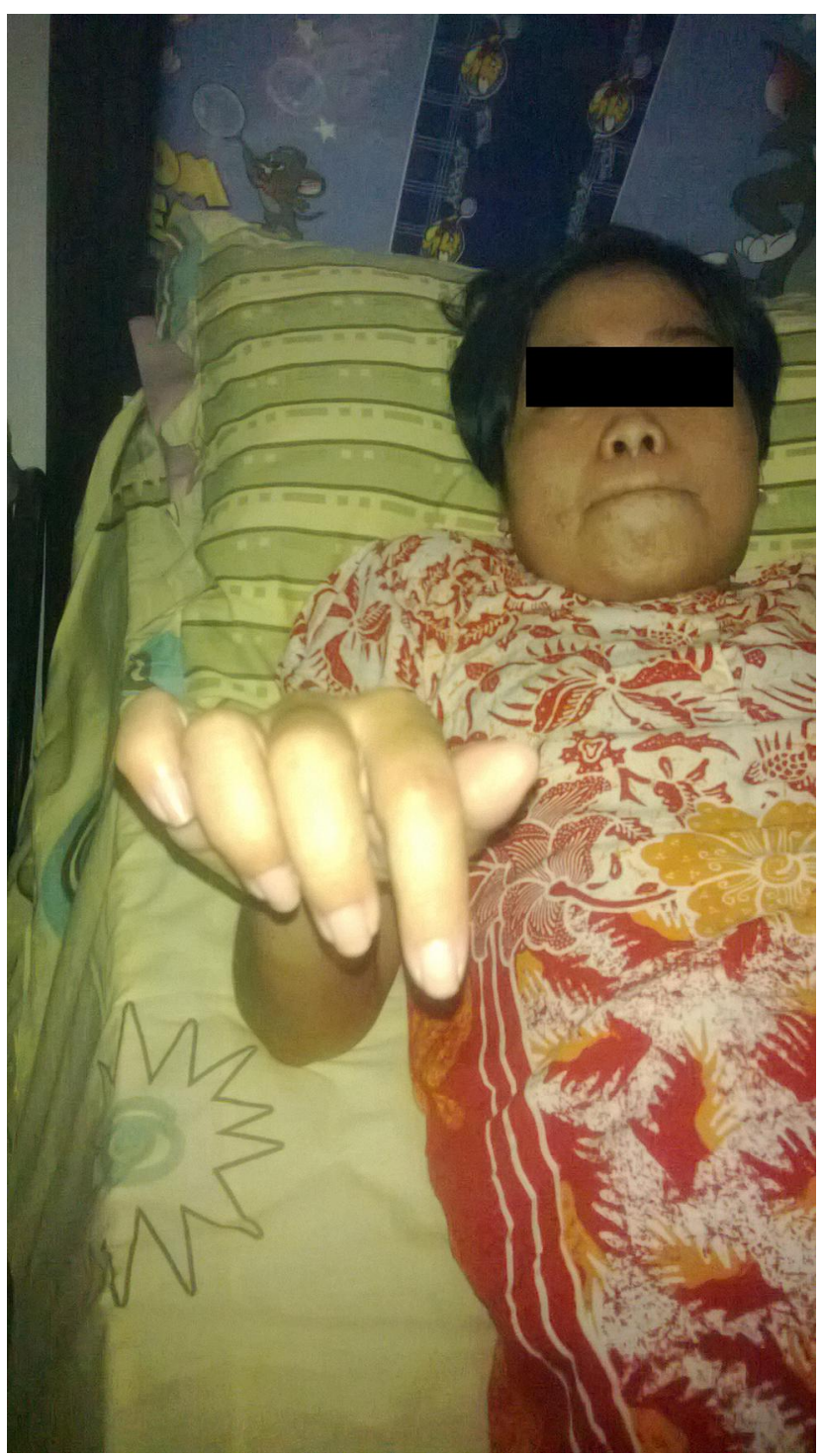

Figure 2 Improvements during hyperbaric therapy on the second day.

and move somewhere while sliding on a wheelchair. During the first seven days, when she moved from the wheelchair to a chair, $80 \%$ of the energy required was derived from a family member who assisted her. On day 10, the patient could raise her arms and legs straight while sitting in a wheelchair (Figure 3).

She was more excited about HBOT therapy. Her appetite was better and a relationship with her surroundings was also pleasant. On day 14 , her family member felt that he only assisted her with $50 \%$ of his energy. The patient started standing on day 18 and was able to walk four steps on day 20 after HBOT (Figure 4).

\section{Discussion}

In general, GBS causes progressive weakness or diffuse paralysis in all four limbs, sensory loss or pain, and 


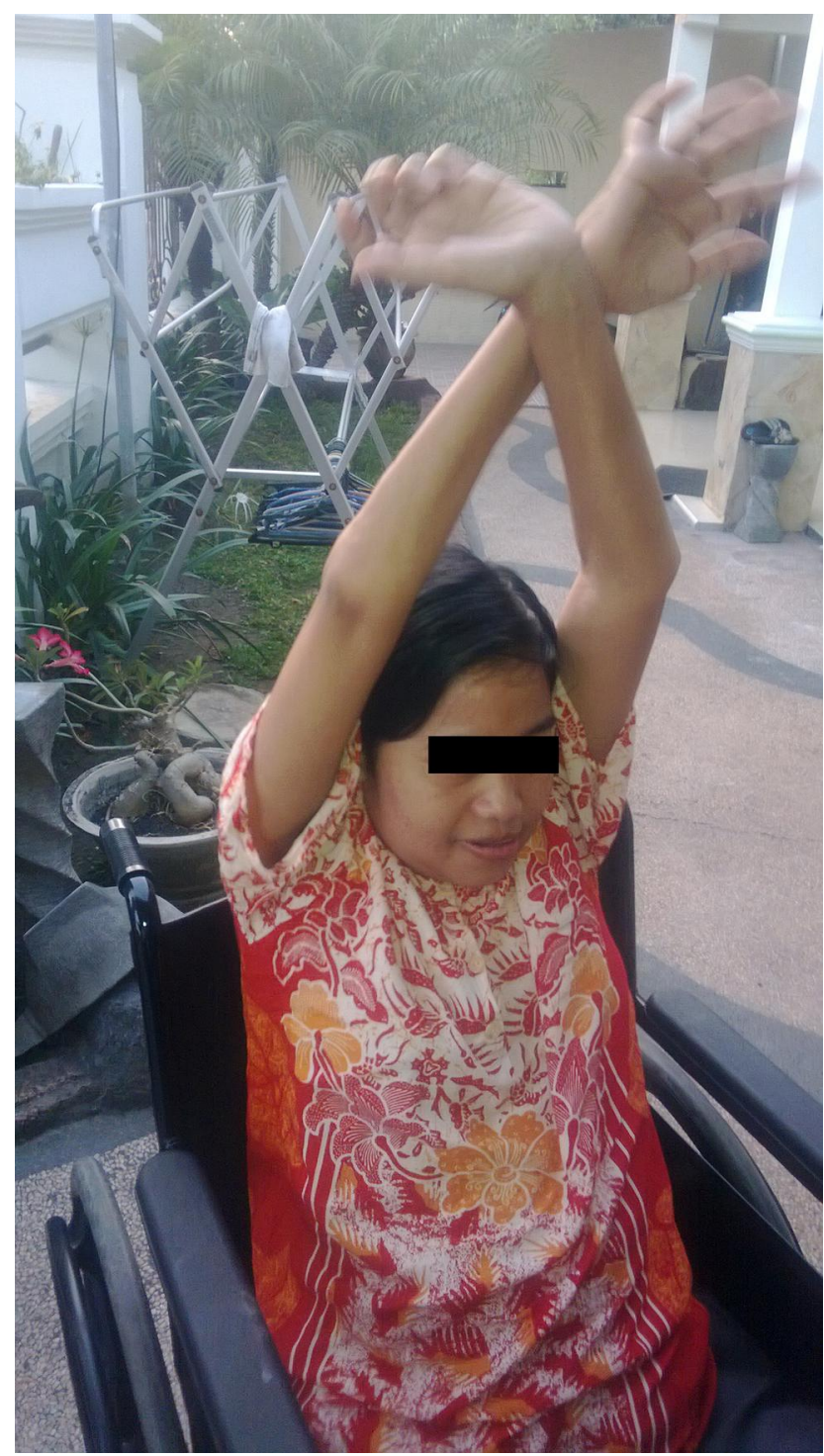

Figure 3 Improvements during hyperbaric therapy on the tenth day.

areflexia. The sudden and unexpected onset of paralysis is very detrimental to patients and is among the most serious emergencies in neurology. ${ }^{4}$ AMAN often follows infection with $C$. jejuni, which contains an epitope in the LOSs that is also present in GM1 gangliosides of nerves. ${ }^{5}$ As a traditional first-line therapy, high-dose IVIG therapy is quite effective in a number of autoimmune disorders by regulating the function of autoreactive T/B cells. ${ }^{6}$ IVIG appears to be ineffective in severe AMAN due to poor modulation of Tfh-B cell interactions. ${ }^{7}$

In some cases, the onset of symptoms is acute, but it is self-limiting with a peak of 2 to 4 weeks and then decreases. ${ }^{8}$ Al-Saffar et al reported a 19 -year-old woman with hepatitis $\mathrm{E}$ infection and bilateral symmetrical weakness, which in turn affected the locomotor muscles.

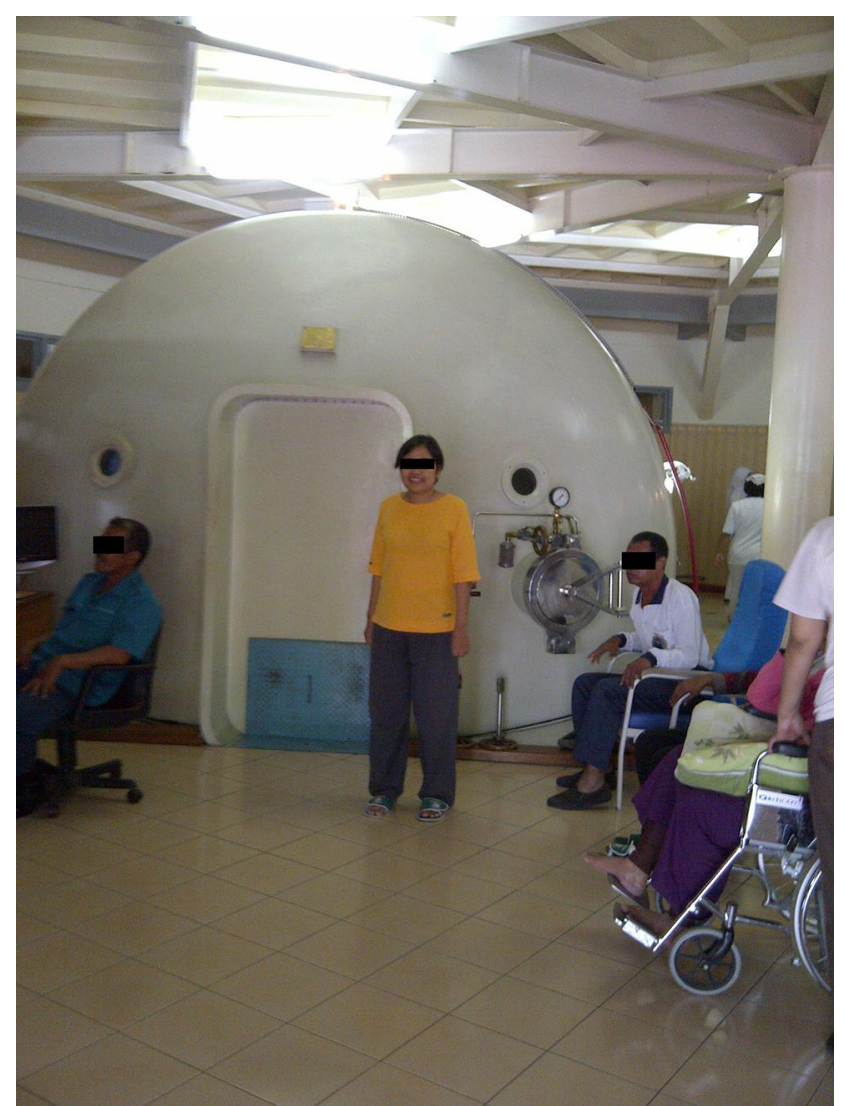

Figure 4 Improvements during hyperbaric therapy on the twentieth day.

Symptoms recovered completely after 35 days with the recovery of liver function. ${ }^{9}$ The opposite occurred in a 32year-old man with tingling fingers after 4 days of taking ciprofloxacin. Popescu recommends folate supplementation for patients with fluoroquinolone-induced neuropathy. Despite the discontinuation of the drug, symptoms persisted. Complete recovery was only achieved after five years. ${ }^{10}$ Propionate is another treatment which was given in the case of a 33-year-old man after receiving IVIG. The regression of this patient's symptoms and improvement in an additional examination appeared to be very good. ${ }^{11}$

Infection may initiate autoimmunity by inducing costimulatory activity in antigen-presenting cells (APC), which is required to induce the development of antigenreactive $\mathrm{T}$ cells, transfer of $\mathrm{T}$ cells to the site of inflammation and the production of inflammatory mediators. ${ }^{4}$ Esposito et al stated that $T$ cells are extensively trained on "self" in the thymus and then move to the periphery, where they seek out and destroy infections and regulate immune response to self-antigens. T cell receptors (TCRs) on $\mathrm{T}$ cells' surface recognize $\mathrm{T}$ cell epitopes, short linear strings of amino acids presented by antigen-presenting 
cells. Some of these epitopes activate $\mathrm{T}$ effectors, while others activate regulatory $\mathrm{T}$ cells. $\mathrm{T}$ cell epitopes that are highly conserved on their TCR face with human genome sequences are often associated with $\mathrm{T}$ cells that regulate immune response. These TCR-cross-conserved or "redundant epitopes" are more common in proteins found in pathogens that have co-evolved with humans than in other non-commensal pathogens. ${ }^{12}$

Inflammation in the nerve roots could lead to disruption of the blood-CNS barrier and allow antibodies against gangliosides to access neural structures near the anterior horn cells, particularly intramedullary collateral branches to the inhibitory interneurons. ${ }^{13}$ Gangliosides (glycancarrying molecules abundantly found in the neural tissues) are expressed as the major antigenic targets for disabilitymediating antibodies. ${ }^{14}$ In AMAN, antibody deposition leads to complement deposition on axolemma, thus damaging the Schwan cell or axon architecture in the nodes and paranodes, in particular leading to disruption of ion channel organization in Ranvier's nodes. Antibody deposition and complement activation increase potassium flow in the paranodic area and decrease sodium flow in the nodes, leading to conduction failure. A generalized disorder of the nervous system in which motor and/or sensory axons are no longer able to communicate effectively between the peripheral and central nervous systems is called nerve degeneration. Channelopathy that occurs in AMAN is one of the mechanisms. ${ }^{15}$

HBO therapy entails inhaling $100 \% \mathrm{O} 2$ with a pressure exceeding atmospheric pressure, given in a high pressure air chamber. The main indications for HBO are decompression sickness, gas poisoning, and gas embolism. ${ }^{16}$ This biological effect results from hypersaturation of the circulating plasma with dissolved oxygen during and immediately after therapy, which results in a momentary increase in the diffusion gradient between the circulation and surrounding tissues. ${ }^{17} \mathrm{HBO}$ therapy with a period of relative hypoxia provides an important oxygen gradient needed to stimulate angiogenesis, fibroblast proliferation, collagen formation, and leukocyte activation which is necessary for tissue repair. ${ }^{18}$

HBOT is reported to improve neurologic recovery after bone marrow injury by ameliorating mitochondrial dysfunction in the motor cortex and bone marrow, arresting the spread of bleeding, reversing the hypoxic process, and reducing edema. ${ }^{19}$ Patel et al who collected various data on HBOT in bone marrow injury, suggested 6 action mechanisms. HBOT reduces apoptosis through various mediators, decreases oxidative stress and fat peroxidation, reduces inflammation, promotes angiogenesis, reduces bone marrow edema, and increases autophagy. ${ }^{20}$

Taking into account the process of infection, inflammation, and autoimmune, HBO can indeed be a rational treatment choice. Patients unable to pay the cost of IVIG or those who have had IVIG and other conventional therapy but have not improved, may undergo HBOT. The clinician shall ensure that the patient has absolutely no contraindications to HBO therapy, that is, an untreated pneumothorax, to prevent an in-chamber emergency.

The concordance of the present case with that described by Song et $\mathrm{al}^{3}$ provides a compelling argument for investigating the role of HBOT in AMAN with larger studies. The similarities to Song's case include: receiving IVIG therapy, experiencing paralysis following HBOT, and improvement after 10 times of HBOT. The difference was that the patient in this case did not undergo mechanical ventilation and only underwent two courses of HBOT; HBOT protocol was 90 minutes at 2.4 ATA with 30 minutes of compression and decompression, MRC on upper limb muscle strength was 5 and lower limb muscle strength was 4, leaving a drop foot. More efforts are needed to explore the effects of HBOT and to demonstrate whether HBO treatment in a wider range of acute inflammatory neuropathies is beneficial. Controlled study data are needed to prove the efficacy of hyperbaric therapy in autoimmune inflammatory neuropathy.

\section{Conclusion}

AMAN is often thought to occur when there are symptoms of Guillain-Barre Syndrome in the productive age. Diagnosis is based solely on the history and physical (internal and neurological) examination. We found a woman diagnosed with GBS who had been undergoing life-saving therapy and receiving conventional therapy. After five days of IVIG therapy with no improvement at all, she underwent HBO therapy. She felt slight daily improvements in the motor muscles of her fingers and toes and in breathing and swallowing. A dramatic improvement also occurred on day 20 when she was able to walk three slow steps without using a walking aid.

\section{Patient's Consent}

The patient and her family gave consent for patient information (photos, videos, and articles) to be published in the journal article. Information will be published anonymously and every effort has been made to ensure anonymity. The 
information will be published in health journals read around the world. Ethical approval and approval of this study include individual patient information stated in the current case report. The approving ethics committee of the revised manuscript was Naval Health Institution.

\section{Acknowledgment}

We would like to thank Lakesla (Naval Health Institute, Surabaya, Indonesia) for providing hyperbaric chamber support.

\section{Author Contributions}

All authors contributed to data analysis, drafting or revising the article, have agreed on the journal to which the article will be submitted, gave final approval of the version to be published, and agree to be accountable for all aspects of the work.

\section{Funding}

No funding or grant support.

\section{Disclosure}

The authors report no conflicts of interest in this work.

\section{References}

1. Estridge R, Iskander M. Understanding guillain-barré syndrome. $J A m$ Acad Physician Assist. 2015;28(7):19-22. doi:10.1097/01.JAA.000 0466585.10595.f5

2. Tamura N, Kuwabara S, Misawa S, et al. Time course of axonal regeneration in acute motor axonal neuropathy. Muscle Nerve. 2007;35(6):793-795. doi:10.1002/mus.20729

3. Song L, Xing B, Yang W, Li H. Hyperbaric oxygen therapy in a patient with guillain-barre syndrome receiving mechanical ventilation. Diving Hyperb Med. 2020;50:306-308.

4. Yang M, Rainone A, Shi XQ, Fournier S, Zhang J. A new animal model of spontaneous autoimmune peripheral polyneuropathy: implications for Guillain-Barre syndrome. BioMedCentral. 2014;2:1-14.

5. Burns TM. Guillain-barré syndrome. Semin Neurol. 2008;28(2):1 52-167. doi:10.1055/s-2008-1062261
6. Sudo M, Miyaji K, Späth PJ, et al. Polyclonal IgM and IgA block in vitro complement deposition mediated by anti-ganglioside antibodies in autoimmune neuropathies. Int Immunopharmacol. 2016;40: 11-15. doi:10.1016/j.intimp.2016.08.019

7. Che Y, Qiu J, Jin T, Yin F, Li M, Jiang Y. Circulating memory $\mathrm{T}$ follicular helper subsets, Tfh2 and Tfh17, participate in the pathogenesis of guillain barre syndrome. Sci Rep. 2016;6:1-14. doi:10.10 38/s41598-016-0001-8

8. Aminzadeh V, Rad AH. A report of guillain-barre syndrome with myalgia and mild weakness. Iran J Child Neurol. 2014;8:70-72.

9. Al-Saffar A, Al-Fatly B. Acute motor axonal neuropathy in association with hepatitis E. Front Neurol. 2018;1-4. doi:10.3389/fneur.20 18.00001

10. Popescu C. Severe acute axonal neuropathy induced by ciprofloxacin: a case report. Case Rep Neurol. 2018;10(2):124-129. doi:10.1159/ 000489303

11. Yoon M, Pitarokoili K, Sturm D, Haghikia A, Gold R, Fisse AL. Treatment of an acute motor and sensory axonal neuropathy with propionate in a 33-year-old-male. Ther Adv Neurol Disord. 2018; 11:1756286418809580.

12. Esposito S, Longo M. Guillain-barré syndrome. Autoimmun Rev. 2017;16(1):96-101. doi:10.1016/j.autrev.2016.09.022

13. Kuwabara S, Yuki N. Axonal guillain-barré syndrome: concepts and controversies. Lancet Neurol. 2013;12(12):1180-1188. doi:10.1016/ S1474-4422(13)70215-1

14. Funes S, Chiari ME, Comín R, Irazoqui FJ, Nores GA. Experimental guillain-barre syndrome induced by immunization with gangliosides: keyhole limpet hemocyanin is required for disease triggering. Biochim Biophys Acta Mol Basis Dis. 2017;1863(6):1473-1478

15. Cashman C, Hoke A. Mechanisms of distal axonal degeneration in peripheral neuropathies. Neurosci Lett. 2015;596:33-50.

16. Jain, Kewal K. Physical, physiological \& biochemical aspects of hyperbaric oxygenation. In: Textbook of Hyperbaric Oxygen. Jain, Kewal K. London: Springer; 2017:11-22.

17. Welslau W. Physiological effects of increased barometric pressure. In: Handbook on Hyperbaric Medicine. Mathieu D. Netherlands: Springer; 2006:31-44.

18. Simsek K, Sadir S, Oter S. The relation of hyperbaric oxygen with oxidative stress - reactive molecules in action. Oxid Antioxid Med Sci. 2015;4:17. doi:10.5455/oams.010415.rv.016

19. Al-Waili N, Butler GJ, Beale J, et al. Hyperbaric oxygen in the treatment of patients with cerebral stroke, brain trauma, and neurologic disease. Adv Ther. 2005;22(6):659-678. doi:10.1007/BF028 49960

20. Patel N, Huang J. Hyperbaric oxygen therapy of spinal cord injury. Med Gas Res. 2017;VII(2):133-140.

\section{Publish your work in this journal}

The International Medical Case Reports Journal is an international, peer-reviewed open-access journal publishing original case reports from all medical specialties. Previously unpublished medical posters are also accepted relating to any area of clinical or preclinical science. Submissions should not normally exceed 2,000 words or 4 published pages including figures, diagrams and references. The manuscript management system is completely online and includes a very quick and fair peer-review system, which is all easy to use. Visit http://www.dovepress.com/testimonials.php to read real quotes from published authors. 